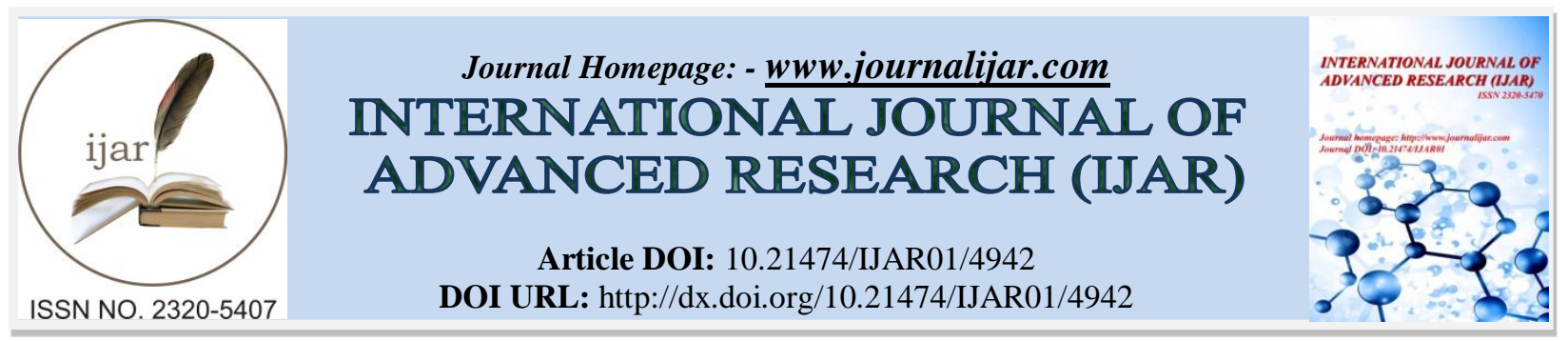

RESEARCH ARTICLE

\title{
CARACTÉRISTIQUES HYDRO SÉDIMENTAIRES DE LA BAIE DE CINTRA (SUD - MAROC).
}

\author{
Ahmed Makaoui ${ }^{1}$, Aziz Agouzouk ${ }^{1}$, Tarik Baibai ${ }^{1}$, Mohammed Idrissi ${ }^{1}$, Jamila Larissi ${ }^{1}$, Ismail Bessa ${ }^{1,2}$, Soukaina Obad $^{12}$, \\ Omar Ettahiri and AlMustafa Ait Chattou et Karim Hilmi ${ }^{1}$. \\ 1. Département d'Océanographie, Institut National de Recherche Halieutique, Casablanca, Maroc. \\ 2. Faculté des Sciences Ben M'Sik, Université Hassan II-Casablanca, Casablanca, Maroc.
}

\section{Manuscript Info}

an......................

Manuscript History

Received: 24 May 2017

Final Accepted: 26 June 2017

Published: July 2017

Key words:-

upwelling, productivity, Cintra Bay, Hydro-Biology, hydrodynamics, Current, bathymetry.

\section{Abstract}

The South Atlantic region of Morocco Dakhla-Oued Eddahab is characterized by a permanent upwelling favouring an abundance of fishery resources and the presence of the Bay of Cintra favourable to aquaculture development. The proximity of these two systems allows them to be permanently influenced by the spatial variability of their marine hydrodynamics.

The South zone is a retention area for mineral enriched waters allowing for high primary productivity of its marine ecosystem. However, intakes of dissolved oxygen-deficient hot water from the south occur exceptionally from one year to the next due to the effect of global warming of the oceans and may have an impact on the ecosystem state of this area. The marine waters of the Atlantic coast are located at the entrance to the Bay of Cintra and can approach the coast at the heart of the bay and to the south by the tidal currents favouring the entry of mineral-rich waters producing an important planktonic richness of the bay.

Thus, the Bay of Cintra is described as a highly potential site for aquaculture regarding its important marine hydrodynamics on the one hand and its enrichment by very productive waters of the Moroccan South Atlantic zone.

Copy Right, IJAR, 2017,. All rights reserved.

\section{Introduction:-}

Cette étude, intégrant les deux aspects (littoral et du large) permettra la mise en place d'un pôle transversal d'océanographie opérationnelle principalement au service de la pêcherie, de l'aquaculture et de l'environnement des sites aquacoles. Ainsi, parmi les sites littoraux marocains, la baie de Cintra présente un grand intérêt pour l'aquaculture. En effet, la région de Oued Eddahab se caractérise par sa proximité á une zone d'upwelling permanant favorisant une abondance en ressources halieutiques et par la présence de la baie de Cintra faisant une superficie de l'ordre de $370 \mathrm{KM}^{2}$ (Longue de $37 \mathrm{Km}$ et large de 10 à $12 \mathrm{Km}$ ) est favorable au développement aquacole. Elle pourrait abriter un observatoire régional d'Océanographie physique pour suivre de prés la variabilité spatiotemporelle du milieu marin de la zone. Et dans le cadre du renforcement des capacités d'observation océanographique de l'INRH dans le milieu marin, la région de Dakhla-Oued-Eddahab a été proposée pour le mouillage d'une bouée océanographique (à partir du 04 octobre 2016) du fait que c'est une zone connue par l'activité de l'upwelling permanant favorisant sa richesse halieutique mais qui a connu un effort de pêche important durant les dernières décennies après l'exploitation des stocks de poissons dans la zone nord atlantique marocaine (FAO, 2010). Cette variabilité des ressources halieutiques peut être aussi tributaire de l'environnement 
océanographique qui dépond énormément de la variabilité climatique. Aussi l'ensemble de l'activité hydrodynamique du large influence l'hydrodynamisme de la zone côtière de la région de Dakhla Oued Eddahab. D'après Makaoui (2008) cette région est subdivisée en trois sous zones de caractéristiques hydrologiques différentes : Sous zone 1 (SZ1): de Boujdor à $25^{\circ} \mathrm{N}$ qui se manifeste par des remontées d'eau profonde côtières dont le centre actif se localise au niveau de la radiale $25^{\circ} 30^{\prime} \mathrm{N}$ et une turbulence importante justifiée par les faibles taux du pigment chlorophyllien; Sous zone 2 (SZ2): de Dakhla à la baie de Cintra caractérisée par une stratification verticale favorable pour la photosynthèse marquée par une richesse en pigments chlorophylliens à la côte de Dakhla et au large de la baie de Cintra. Aussi des eaux fraîches loin de la côte se manifestent au niveau de la radiale $23^{\circ} 30^{\prime} \mathrm{N}$ et semblent due à une dérive des eaux de résurgences vers le large qui peuvent expliquer l'abondance élevée des zooplancton détecté au large de Dakhla (Somoue et al., 2003) ; Sous zone 3 (SZ3): de cap Barbas au cap Blanc marquée par une stratification qui se manifeste par la présence de différentes masses d'eau et une advection des Eaux Centrales Sud Atlantiques (ECSA) riches en nutriment du sud vers le nord caractérisée par la présence d'un front thermo - halin au niveau du cap Blanc.

L'objectif principal de cette étude s'articule sur la description de l'hydrodynamique de la baie de cintra et ses caractéristiques océanographiques et leur influence par les résurgences d'eau profonde de la zone littorale de Dakhla - Oued Eddahab dans le but de développer une activité aquacole au niveau d'un site potentiel de cet écosystème.

\section{Méthodologie:-}

Deux campagnes océanographiques ont été réalisées dans la baie de Cintra à bord du Zodiac Dauphin I du CRINRH-Dakhla pour l'étude hydro biologique et hydrodynamique de son écosystème marin (Octobre 2015 et Mai 2016). Le réseau d'échantillonnage comporte treize stations océanographiques et soixante levées bathymétriques présentées selon le réseau sur la figure 1:

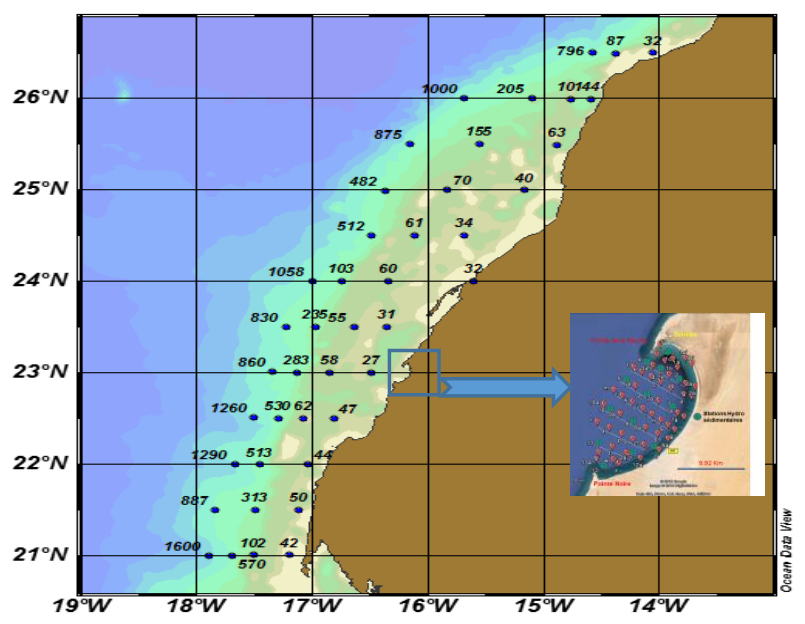

Figure 1:- Carte du réseau d'échantillonnage des stations océanographiques des zones d'étude (Zone sud : Cap Boujdor $26^{\circ} \mathrm{N}$ - Cap Blanc $21^{\circ} \mathrm{N}$ et baie de Cintra

Les actions réalisées pendant les campagnes dans la baie de Cintra sont:

- Le prélèvement des paramètres physiques (température, salinité, oxygène dissous) par une multi sonde CTD Hanna (HI9828 Multiparameter Water Quality Meter) et des échantillons d'eau filtré au laboratoire des ressources littorales de l'INRH à Dakhla pour la détermination de la matière en suspension (MES). L'analyse des éléments nutritifs (nitrates, nitrites, Phosphates et silicates) ont été réalisées au Laboratoire d'Océanographie Physique de l'INRH-Casablanca par un Auto analyseur de type AA3 AxeFlow;

- La collecte de 13 échantillons de sédiment pour l'étude de la MO et des faciès de la baie par une benne de type Van Veen de petite taille ;

- Le mouillage d'un courantomètre Aandera RCM9 au niveau de la baie de Cintra à $5 \mathrm{~m}$ de la surface (Station 9) : Mis à l'eau le 11/10/2015 à 12 H 15 et récupéré le 20/10/2015 à 14 H;

- La collecte des levés bathymétriques pendant la mission du mois d'octobre par la sonde Eagle et le GPS map 62 (Garmin) du Laboratoire d'Océanographie Physique au niveau du réseau bathymétrique présenté sur la figure 1. 
- Les paramètres collectés et les échantillons conservés durant la campagne sont traités aux laboratoires d'océanographie Physique de l'INRH - Casablanca.

Pendant la même période (Octobre 2015 et mai 2016), des campagnes océanographiques ont été réalisées en automne et en été, le long du littoral de la zone comprises entre Cap Boujdor $\left(26^{\circ} \mathrm{N}\right)$ et Cap Blanc $\left(21^{\circ} \mathrm{N}\right)$. Les paramètres hydrologiques (Température, Salinité, Oxygène dissous et Phosphates) ont été prélevés au niveau des différentes stations répartie le long du littoral de la zone d'étude selon un réseau présenté sur la figure 1.

\section{Resultats et Discussion:-}

\section{III-1. Hydro climat de la zone littorale:-}

D'après les statistiques des vents mesurés à Dakhla, les vents dominants sont NNE et ENE. Pour les autres directions, qui se manifestent surtout en automne, l'intensité du vent est faible. Ces vents dominants peuvent avoir un rôle d'engraissement de la baie par les apports éoliens principalement du côté nord de la baie abritée par la pointe nord. Concrètement, la zone d'étude se caractérise par un vent fréquent et régulier, les vents dominants sont donc les alizés continentaux du NE qui peuvent être associés aux alizés océaniques du NO, chargés d'humidité et plus frais. Les vents de l'Est soufflent localement en hiver. En provenance du Sahara, ces vents secs et chauds apparaissent lorsque les alizés sont presque nuls.

Les vents sont de force moyenne, d'une vitesse proche qui va de 6 à $10 \mathrm{~m} / \mathrm{s}$. Chaque année des vents supérieurs à 16 $\mathrm{m} / \mathrm{s}$ sont enregistrés entre 120 et 160 jours. La période estivale, allant de juin à août, est la plus forte. La vitesse moyenne des vents plus forts sont comprises entre 20 et $21 \mathrm{~m} / \mathrm{s}$ (ANDA, 2015).

Les travaux de SOGREAH $(1983,1984)$ indique que l'origine de la houle au large de Oued - Eddahab se trouve dans les perturbations du milieu de l'Atlantique. Ces dernières sont en relation avec la circulation atmosphérique qui prévaut sur la partie Nord-Est de l'océan atlantique. La houle présente les mêmes caractéristiques que le vent: une régularité tout au long des saisons. Ce sont des vagues d'origine locale ou lointaine, d'amplitude modérée. Les directions dominantes de la houle en long s'inscrivent dans le secteur N350 à N040 (plus de $70 \%$ de la houle). La houle se propage vers la côte. Au cours de la propagation, les vagues se déforment à cause de l'effet du fond. La houle est le principal phénomène responsable de l'évolution du littoral; il est donc important de déterminer ces modifications. L'étude de la houle dans les profondeurs indéfinies caractéristiques de la zone de Cintra inclut la caractérisation du régime moyen de la houle définie par la distribution statique des différents états de la mer (ANDA, 2015). Le tableau présenté ci-dessous (Tableau 1), et qui fait référence à la hauteur de vague significative, offre la série de résultat suivante :

$>$ La série temporelle inclut trois évènements qui dépassent les 5 mètres de hauteur de vague, dont un évènement dépasse les 6 mètres de hauteur de vague significative (ANDA, 2015).

$>$ Les houles du nord-nord-ouest et du nord sont celles qui ont une fréquence de présentation plus élevée. De celles-ci, celles du nord-nord-ouest sont celles qui représentent plus de $25 \%$ (ANDA, 2015).

$>$ Le tableau des statistiques de base démontre numériquement la probabilité de fréquence de la direction nordnord-ouest est de 28,9\% et la Hs 12 de 4,49 mètres. La probabilité de fréquence de la direction nord est de $25 \%$ et la Hs12 de 3,49 mètres, alors que la fréquence du nord-ouest est de $15 \%$ et la Hs12 de 4,9 mètres. 
Tableau 1:- Statistiques de base de la période moyenne de la vague dans les eaux profondes indéfinies (ANDA, 2015).

\begin{tabular}{|c|c|c|c|c|c|}
\hline Direction & Prob.direction & $\mathrm{Hs}_{5090}$ & $\mathrm{Hs}_{9090}$ & $\mathrm{Hs}_{9996}$ & $\mathrm{Hs}_{12}$ \\
\hline $\mathbf{N}$ & 0,2577 & 927 & 7958 & 10764 & 19852 \\
\hline NNE & 0,0391 & 598 & 5877 & 5904 & 12478 \\
\hline $\mathrm{NE}$ & 0,0028 & 477 & 2582 & 744 & 3856 \\
\hline ENE & 0,0007 & 269 & 735 & 39 & 1073 \\
\hline $\mathbf{E}$ & 0,0001 & 293 & 456 & 35 & 829 \\
\hline ESE & 0,0001 & 120 & 222 & 19 & 390 \\
\hline SE & 0,0000 & 111 & 101 & 10 & 255 \\
\hline SSE & 0,0000 & 85 & 48 & 6 & 167 \\
\hline $\mathrm{S}$ & 0,0000 & 157 & 192 & 14 & 387 \\
\hline SSW & 0,0000 & 109 & 176 & 23 & 341 \\
\hline SW & 0,0001 & 159 & 289 & 12 & 494 \\
\hline WSW & 0,0003 & 237 & 277 & 16 & 573 \\
\hline W & 0,0020 & 334 & 341 & 15 & 730 \\
\hline WNW & 0,0211 & 264 & 269 & 14 & 576 \\
\hline NW & 0,1493 & 334 & 854 & 60 & 1276 \\
\hline NNW & 0.2891 & 484 & 4375 & 1651 & 6556 \\
\hline Total & 797 & 4958 & 24752 & 19326 & 49833 \\
\hline
\end{tabular}

Cette zone est caractérisée principalement par l'existence du courant variable principalement entre la direction sudouest à sud-est (à 10m de la surface) et d'une intensité maximale de l'ordre de 34 à $40 \mathrm{~cm} / \mathrm{s}$ et par le développement des filaments marqué en surface principalement en printemps - été (Makaoui, 2008). Ces filaments sont provoqués par des courants côtiers chauds au niveau des baies d'Agadir (Hagen et al., 1996; Pelegri et al., 2005a, Makaoui et al., 2012) (Minas et al., 1982) et de Dakhla (Wauthy, 1983) et se dirige au nord provoquant la dérive des eaux froides vers le large. Le flux de surface, normalement en direction de l'Equateur, est également inverse entre les iles Canaries et le Maroc à la fin de l'automne et en hiver, probablement en raison d'un affaiblissement des alizes au sud du Cap Ghir et à Dakhla. L'influence du contre-courant Nord-Equatorial s'intensifie durant la saison estivale et le courant des Canaries ne pénètre pas au-delà de $15^{\circ} \mathrm{N}$ au sud et de $20^{\circ} \mathrm{W}$ à l'Est (Barton et al., 1998). Cette structure est favorisée au niveau de la zone sud par une bathymétrie caractérisée par une configuration du plateau continental peu profond et très large. Les faibles profondeurs se présentent de part et d'autre de Dakhla et les plus fortes profondeurs s'approche de la zone de Dakhla du nord vers le sud au niveau de la baie de Cintra. L'étude granulométrique des sables prélevés dans la zone montre généralement la présence d'un seul faciès sédimentaire sableux quasiment dans toute la zone. Dans ce faciès, plus de $95 \%$ de ses éléments sont des arénites représentées généralement par des sables grossiers à très grossier. Ainsi, la zone nord de Dakhla, où se manifeste l'upwelling de cap Boujdor, l'hydrodynamique est élevé, visualisée par la dominance de la fraction grossière ; Cependant au niveau de la zone comprise entre Dakhla et la baie de Cintra, marquée par une stratification hydrologique verticale, la turbulence décroît, marquée par la présence du sable moyen et du sable fin. Ce qui explique la diminution de l'hydrodynamique de la zone sud Dakhla par apport au nord (Makaoui, 2008). Ces deux facteurs (Upwelling et filament) contribuent á la haute productivité de cette zone et par suite vont contribuer à l'influence des résurgences d'eau profonde sur la richesse de l'écosystème côtier de la région de Dakhla - Ouesd Eddahab notamment les baie de cintra et de Dakhla. En termes de variabilité interannuelle sur la période 2002-2014, l'activité de l'upwelling, évaluée par un Indice d'Upwelling Côtier (Coastal Upwelling Index) hebdomadaire (Benazzouz et al., 2013, 2014) basé sur les images satellitaires de la SST, présente généralement une variabilité annuelle marquée par une tendance vers la baisse durant les années 2004-2007, 2009-2010 dénotant une faible activité de l'upwelling et vers la hausse sur la période 2011 et 2014, soit une forte activité du phénomène. 
Dans la région de Cintra, la marée est semi-diurne et son niveau moyen est de $+1,30 \mathrm{~m}$ par-dessus du zéro hydrographique. Le tableau ci-après (tableau 2) présente les niveaux des marées à l'intérieur de la baie (ANDA, 2015).

Tableau 2:- Les données de Marées (ANDA 2015).

\begin{tabular}{|c|c|c|c|c|}
\hline & \multicolumn{2}{|c|}{ Équinoxe (m) } & \multirow{2}{*}{$\begin{array}{l}\text { Moyenne des } \\
\text { Marées de } \\
\text { Vive-Eau (m) }\end{array}$} & \multirow{2}{*}{$\begin{array}{l}\text { Moyenne des } \\
\text { Marées de } \\
\text { Morte-Eau } \\
\text { (m) }\end{array}$} \\
\hline & Printemps & Automne & & \\
\hline Pleine mer & $+2,40$ & $+2,60$ & $+2,30$ & $+1,70$ \\
\hline Marée Basse & $-0,20$ & $+0,20$ & $+0,30$ & $+0,90$ \\
\hline
\end{tabular}

Le long de la côte atlantique marocaine, l'onde de marée est de type semi-diurne et d'amplitude variable, elle vient du Sud entraînant ainsi un flot vers le large. Les enregistrements de marée mesurés au niveau du port de Dakhla montrent que le marnage varie entre 0.5 et $2.5 \mathrm{~m}$ et en fonction du coefficient de marée des mortes eaux et des vives eaux (Orbi et al., 1995). Pendant la période d'automne (Octobre), nous pouvons constater sur le graphique des horaires des marées (fig. 2) que la première basse mer s'est produite à $5 \mathrm{~h} 20$ et la suivante à $17 \mathrm{~h} 35$. La première pleine mer s'est produite à $11 \mathrm{~h} 35$ et la suivante à $23 \mathrm{~h} 50$. Le coefficient de marée a été de 83 (haut). Les hauteurs des marées ont été de $0,5 \mathrm{~m}$, de $2,3 \mathrm{~m}$, de $0,4 \mathrm{~m}$ et de $2,3 \mathrm{~m}$. Nous pouvons comparer ces niveaux avec la plus haute pleine mer enregistrée sur les tables de marées à Dakhla qui est de 2,8 $\mathrm{m}$ avec une hauteur minimale de $-0,3 \mathrm{~m}$. (http://www.mareespeche.com). La majorité des stations océanographiques ont été réalisées en pleine mer et en vives eaux.
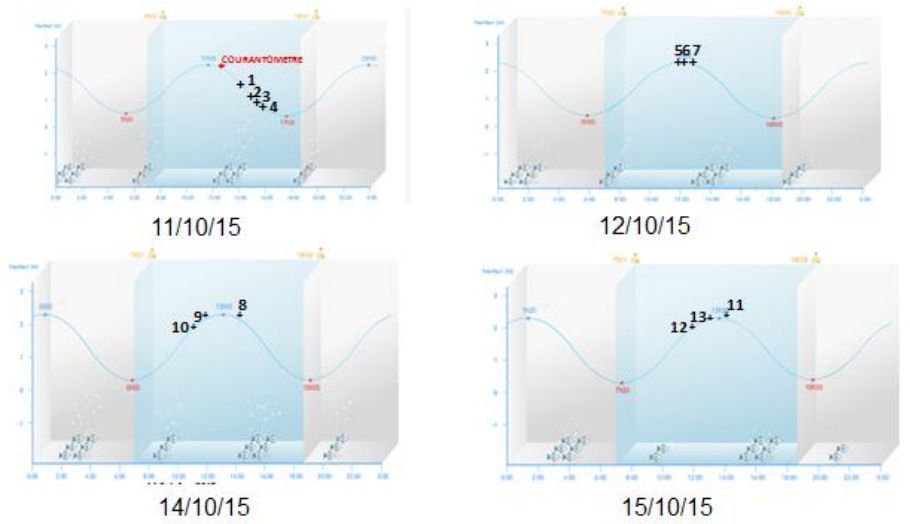

Figure 2:- Les horaires des marées et des stations océanographiques à Cintra (du 11 au 20 Octobre 2015)

Pendant la période de printemps (Mai), nous pouvons constater sur le graphique des horaires des marées que la première basse mer s'est produite à $8 \mathrm{~h} 15$ et la suivante à $20 \mathrm{~h} 45$ (fig. 3). La première pleine mer s'est produite à $14 \mathrm{~h} 35$ et la suivante à $15 \mathrm{~h} 20$. Le coefficient de marée a été de 100 (très haut). Les hauteurs des marées ont été de $0.1 \mathrm{~m}$ et $2,5 \mathrm{~m}$ et c'est la plus haute pleine mer enregistrée sur les tables de marées à Dakhla (http://www.mareespeche.com). La majorité des stations océanographiques ont été réalisées au début de la pleine mer et en vives eaux. 


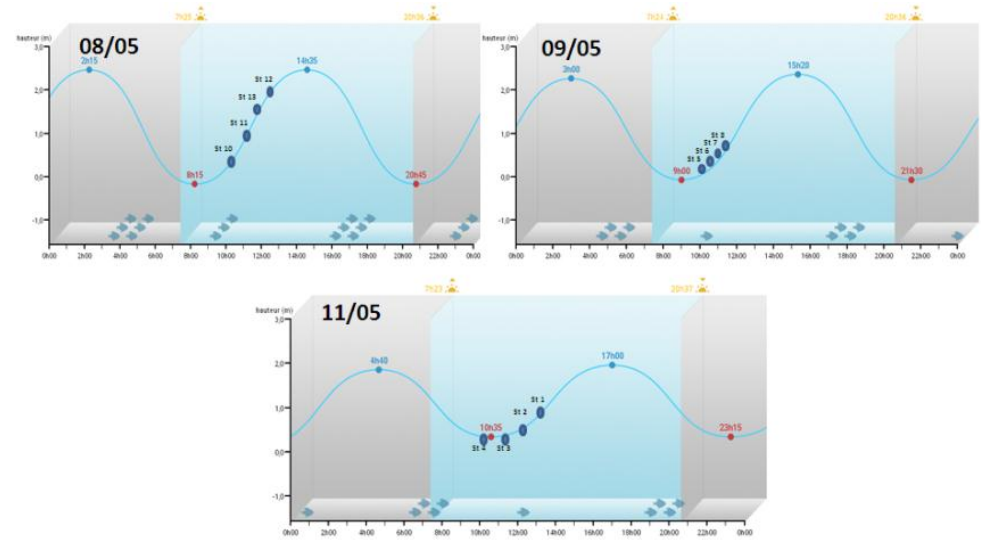

Figure 3:- Les horaires des marées et des stations océanographiques à Cintra (du 08 au 11 mai 2016)

Cependant, les principaux courants observés dans la zone d'étude sont des courants de marée, à haute mer et à basse mer. Le courant de haute mer suit la direction est dans le passage et la direction nord-est dans le canal vers le nordest. Le courant de mer basse suit la direction ouest dans le passage et la direction sud-ouest dans le canal. La baie de Cintra se caractérise surtout par des courants plus intenses (ANDA, 2015).

Le courant a été observé et mesuré à une profondeur de $5 \mathrm{~m}$ de la surface à la station 9 située au centre de la baie de Cintra sur une dizaine de jours environ du 11 au 20 Octobre 2015. Sur cette période de mesures, elle correspond à une période intermédiaire comprise entre des marées de vive eau et de morte eau (figure 4a), correspondant à des coefficients de marée compris entre 58 et 80 . Sur cette période d'observation, le marnage est de l'ordre de $2.5 \mathrm{~m}$ environ en période de vive eau et de $0.8 \mathrm{~m}$ environ en période de morte eau (Figure $4 \mathrm{~b}$ ). S'agissant du courant, on constate que son intensité varie entre $0.3 \mathrm{~cm} / \mathrm{s}$ et $20 \mathrm{~cm} / \mathrm{s}$, avec une moyenne de $8.1( \pm 3.75) \mathrm{cm} / \mathrm{s}$ sur cette période d'observation. En étant soumise à l'influence des marées, les directions du courant dans la baie montrent globalement des courants de marée dans une direction Nord Nord Est (flux) pendant la marée haute à Sud Sud Ouest (reflux) pendant la marée basse sur cette période d'observation. En effet, des sorties du modèle 2D des exemples de configuration de la circulation de cette baie selon la situation en période de marée de vive eau en prenant l'exemple de la journée du 17 octobre 2016 à $01 \mathrm{~h}$ à marée entrante coïncidant avec des vents de secteur Nord Est (Figure 5), les courants sont orientés du Nord au Sud avec des intensités comprises entre $0.1 \mathrm{~m} . \mathrm{s}-1$ et $0.5 \mathrm{~m} . \mathrm{s}-1$. Les plus forts courants sont observés au Nord du domaine d'étude et au large de la baie. A l'intérieur de la baie, les courants sont dirigés du Nord au Sud mais avec des intensités de moindre amplitude (< à 0.3 m.s-1) (Figure 5).

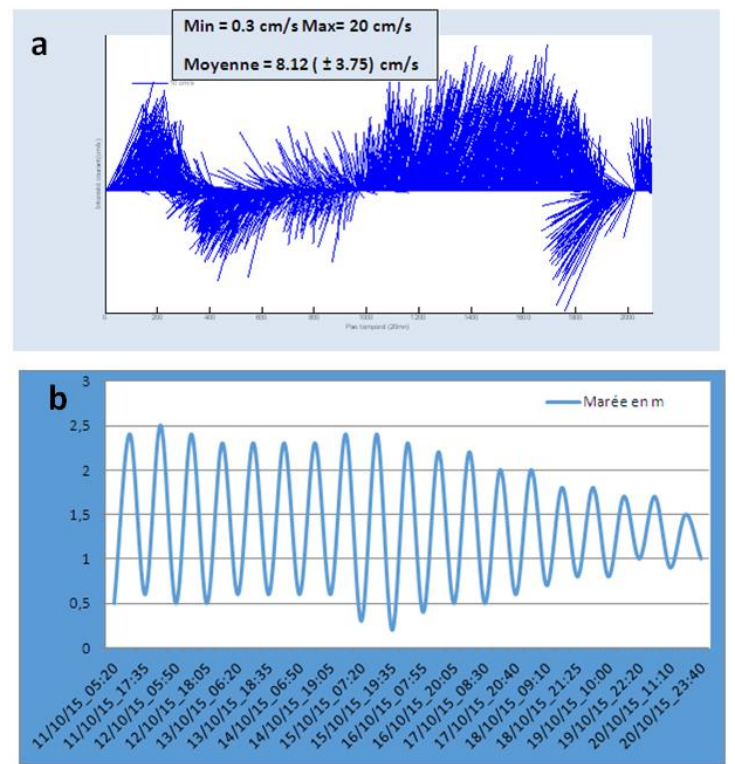

Figure 4:- Intensité du courant dans la baie de Cintra (du 11 au 20 Octobre 2015) 

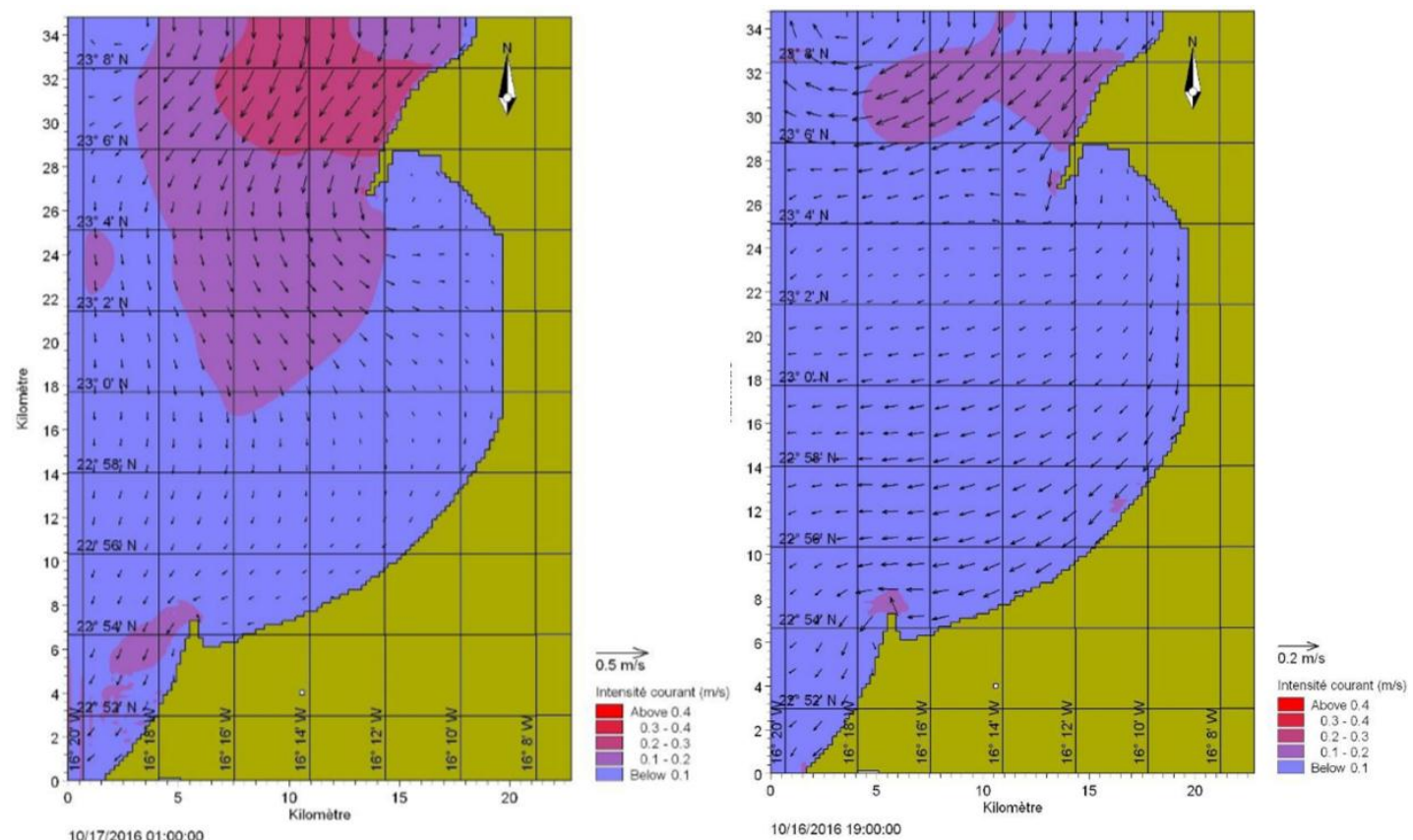

Figure 5:- Exemple de circulation de la baie de Cintra en période de vive-eau à marée entrante (à gauche) et sortante (à droite) (Hilmi et al., 2017).

En effet, la baie est assez ouverte, à caractère sableux, qui contient de nombreuses dunes de faible hauteur, limitée par la pointe de las Raimas au nord et la pointe Negra au sud. Elle est donc très influencée par les conditions hydrodynamiques du littoral atlantique de la région.

L'extrémité nord, est une péninsule de sable inondable de 2 milles d'extension vers le sud-ouest, se terminant sur des pierres sableuses et un récif naturel. L'extrémité sud, est couronnée par une colline de sable elle aussi entourée de récifs qui se prolongent environ de moins de 2 milles vers le nord-est. Le littoral est constitué par un massif et des dépôts de sable subjacent. Toute la baie de Cintra possède la même structure géologique. On observe uniquement une différence de hauteur des deux côtés de la baie, résultat de l'effondrement de la péninsule par rapport au bassin Aguerguer. La péninsule est unie au continent par un isthme formé par des matériaux tertiaires et des sédiments quaternaires actuels qui couvrent les dépôts tertiaires sur des hauteurs de 20 mètres. Le diamètre moyen des sédiments dans la région est de l'ordre de 0,2 à $0,3 \mathrm{~mm}$ et la dureté (le contenu calcaire) entre 20 et $30 \%$, ce qui correspond à des sables moyens typiques du dynamisme sédimentaire de la côte atlantique marocaine (ANDA2015).

A l'extérieur de la baie, la bathymétrie est irrégulière avec des différences de profondeurs qui peuvent être importantes. Au niveau des pointes Nord et Sud, on trouve la ligne bathymétrique $-15 \mathrm{~m}$ très proche de la côte. Au niveau de la pointe Nord (pointe de Glab), la pente des fonds vers le large est de $0,2 \%$. La ligne bathymétrique -20 $\mathrm{m}$ se trouve à $10 \mathrm{~km}$ de la côte. Dans l'Ouest de la pointe Sud (pointe Négra), la pente des fonds vers le large est de 1,5\%: la ligne bathymétrique $-15 \mathrm{~m}$ se trouve à $2 \mathrm{~km}$ de la côte. Au niveau de la pointe nord, une partie du terrain est située au-dessous du niveau de la mer (zone inondable) (DPDPM - Annuaire des côtes marocaines, province de Dakhla, 2013).

A l'intérieur de la baie, la profondeur est plus faible (Makaoui et al., 2016). La carte bathymétrique de la baie, réalisée principalement à marée haute, est corrigée par rapport aux données SHOM (Site web (www.shom.fr)) indique une faible variation de la profondeur à l'intérieur de la baie. La majeure partie du bassin a une profondeur allant de -12 à $-10 \mathrm{~m}$. Les faibles profondeurs sont plus proches de la côte où le plateau de $-10 \mathrm{~m}$ à la côte $(0 \mathrm{~m})$ est très court avec une pente plus importante à l'exception de la zone nord abrité par la pointe nord où le plateau est plus large et peu profond en s'allongeant vers la sebkha située au nord de la baie (fig. 6). 


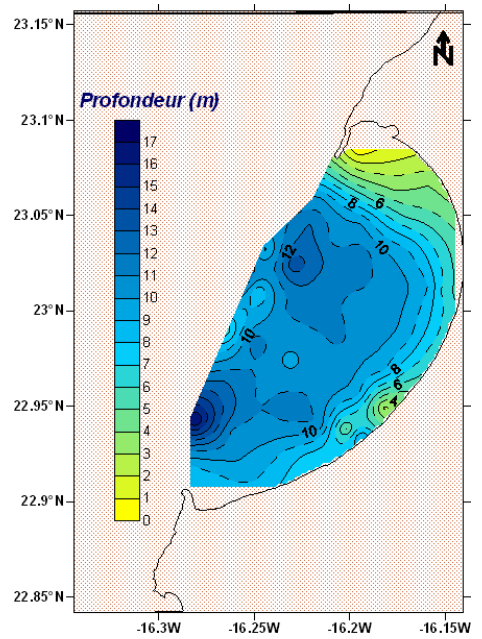

Figure 6:- Carte bathymétrique dans la baie de Cintra (Octobre 2015)

Au nord et au centre de la baie, le sédiment est constitué généralement de sable fin à moyen. Par contre la partie sud de la baie, le sédiment est majoritairement constitué de sables grossiers et très grossiers. Ce qui montre que la baie est plus abritée au nord et au milieu qu'au sud qui est exposé à la houle provenant du nord-ouest de la baie.

L'étude granulométrique du sédiment prélevé en mois d'octobre, au niveau de la baie de Cintra montre la présence de deux faciès sédimentaires; un faciès sableux et un faciès sable vaseux. L'analyse granulométrique nous a permis de mettre en évidence deux secteurs morpho - sédimentaires au niveau de la baie. Un secteur au nord abrité par le cap de la pointe de las Raimas caractérisé par une faible hydrodynamique marine marquant le phénomène de la dissipation de la houle (perte d'énergie). Un deuxième secteur caractérisé par une forte hydrodynamique marine au large et au sud de la baie qui est provoqué par l'intensité de la houle, surtout que cette dernière résulte d'un champ de vent Nord à Nord Est et dépend de l'enfoncement de sédiment dans l'eau qui provient de l'embouchure torrentiels ou de l'érosion des falaises, car plus les sédiments s'enfoncent plus la houle sera grande (Fig. 7).

L'analyse de la matière organique au niveau de la baie de Cintra, montre un gradient + /- homogène. Les teneurs varient entre $0.45 \%$ et $3.2 \%$. Au niveau de l'amont de la baie de Cintra, on enregistre des teneurs $+/$ - homogènes à l'exception de la station 4 située à proximité de Punta de Las Raimas qui marque des teneurs élevées de la matière organique. Au centre de la baie, les teneurs de la matière organique sont faibles à très faibles qui ne dépassent pas (1\%) où on a l'existence des sédiments grossiers à cause de la présence d'une forte agitation provoquée par un mouvement giratoire des courants en fond et qui ne permet ni la sédimentation de la fraction fine ni la sédimentation de la matière organique. Cette répartition des taux de la matière organique est tout à fait conforme avec l'organisation générale de l'écosystème et le fonctionnement de la baie, notamment en ce qui concerne son hydrodynamique. 

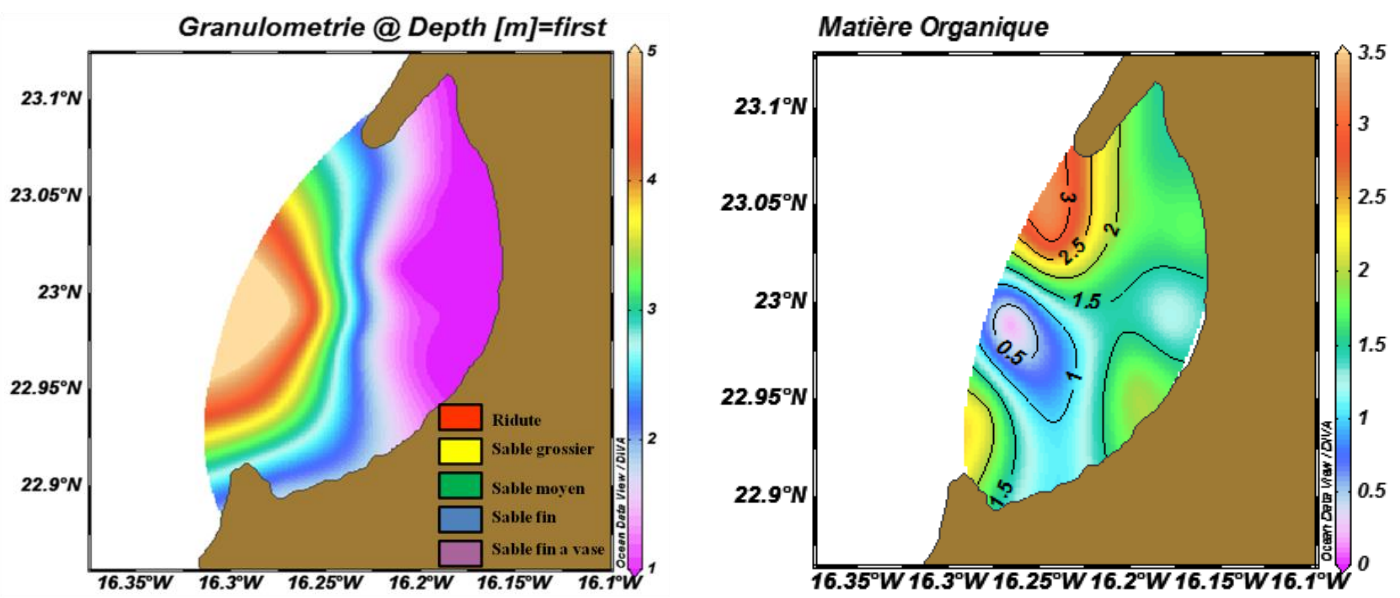

Figure 7:- Répartition granulométrique et de la matière organique des sédiments dans la baie de Cintra (Octobre 2015)

La distribution des paramètres physiques (température ${ }^{\circ} \mathrm{C}$, Salinité psu) au niveau de la baie indique une variabilité saisonnière marquée par des eaux plus chaude en octobre qu'en mai. Par contre la distribution spatiale se manifeste par l'existence de deux masses d'eau réparties en aval et en amont de la baie. Une masse d'eau chaude, plus salée localisée au nord de la baie et répartie sur toute la côte et au fond de toute la baie et une masse d'eau superficielle à l'entrée de la baie de caractéristique marine d'une salinité relativement faible de l'ordre de 36.25psu mais relativement froide. En effet, les eaux marines se localisent à l'entrée de la baie et s'approchent de la côte au niveau du cœur de la baie. Alors qu'au nord et au sud, les eaux marines affrontent des eaux littorales chaudes et plus salées localisées dans la zone nord et longeant toute la côte de la baie marquant ainsi un front thermo halin plus marquée au nord qu'au sud (fig. 8). 


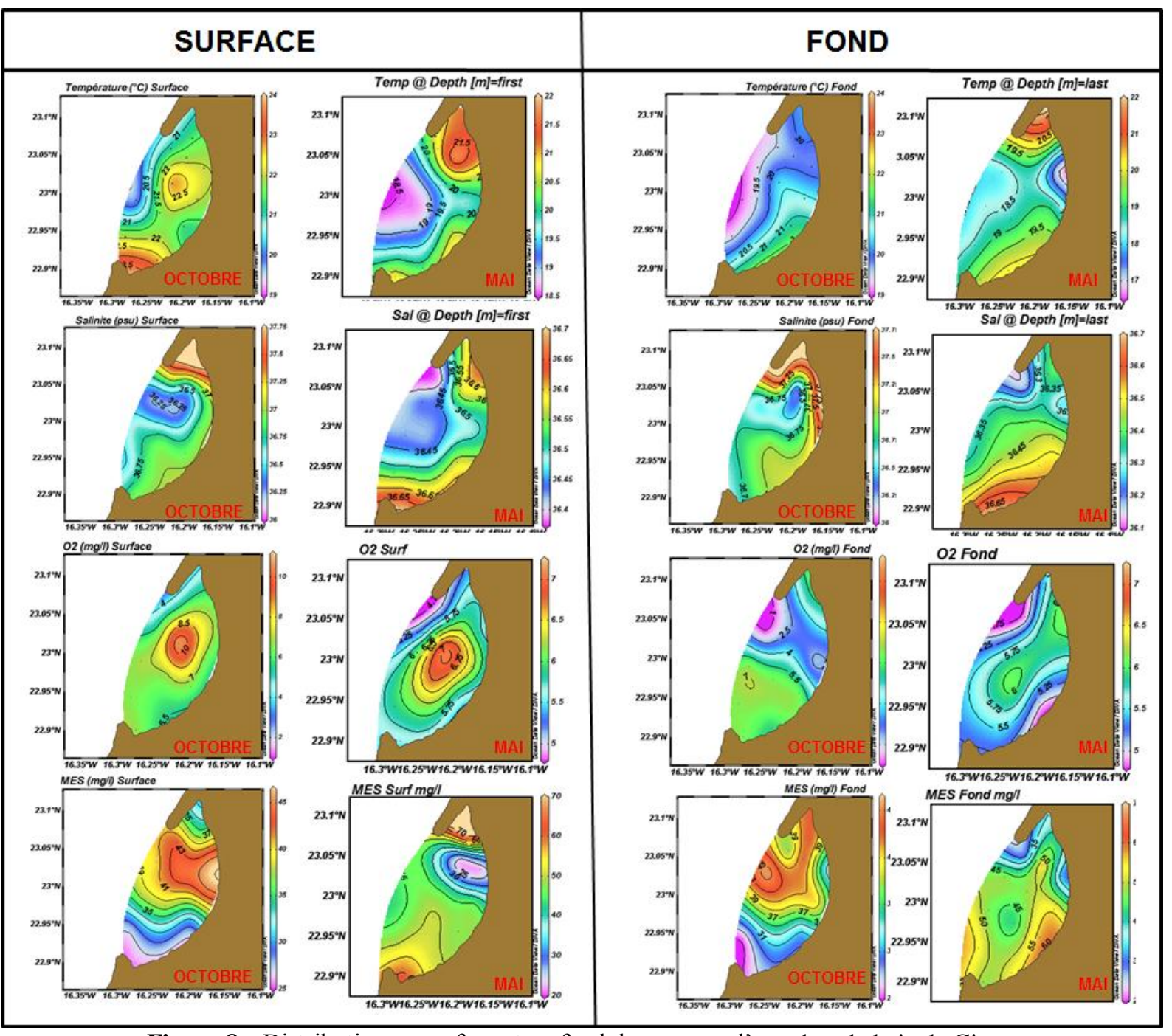

Figure 8:- Distribution en surface et au fond des masses d'eau dans la baie de Cintra

(Octobre 2015 et Mai 2016)

La distribution des teneurs en oxygène dissous en surface indique que la masse d'eau entrante occupe toute la baie et elle est plus saturée en oxygène marquée par des teneurs de l'ordre de $8.5 \mathrm{ml} / 1$ en octobre et $7 \mathrm{ml} / 1$ en mai. Alors qu'au fond, les eaux situées au nord de la baie, zone abritée et en contact direct avec la sebkha, sont moins saturées que les eaux situées au sud qui sont aussi plus saturées que les eaux de la masse d'eau entrante dans la baie. Cette zone moins saturée en oxygène dissous se manifeste par une turbidité importante marquée par les quantités élevées de la MES principalement en surface (fig. 8).

Les distributions des nutriments le long de la baie de cintra indiquent que la zone nord, du côté de la pointe du nord, se manifeste par une richesse importante en nitrates et en phosphates aussi bien au fond qu'à la surface qui se manifeste par des concentrations respectivement de l'ordre de 6 à $8 \mu \mathrm{M}$ et 05 à $1.5 \mu \mathrm{M}$ en mois d'octobre 2015 (fig. 9). Alors qu'en mois de mai 2016, les concentrations enregistrées sont légèrement moins importantes mais elles présentent la même répartition aussi bien au fond qu'à la surface. Cette richesse minérale peut être causée par l'envahissement des eaux marines du littoral et par les anciennes habitations du village de pêche qui occupaient la zone (Voir photo 2) depuis longtemps jusqu'à l'année 2008, et que les traces existent encore sur le sédiment du site. Aussi sa richesse en silicium pendant les deux périodes de l'étude traduit bien l'influence éolienne qui alimente en continu la baie en grains de sable continentaux. 


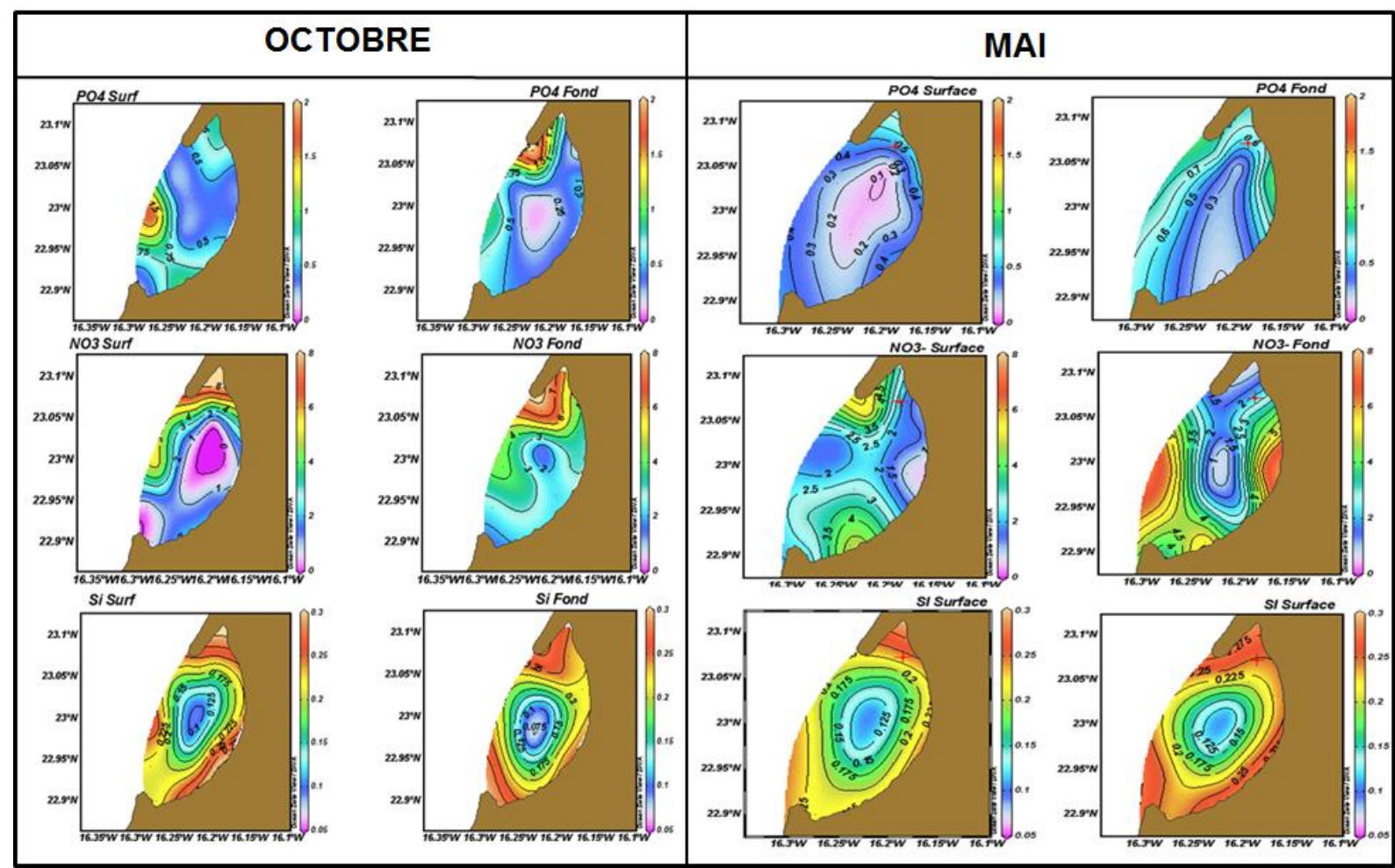

Figure 9:- Distribution en surface et au fond des nutriments (PO4, NO3 et $\mathrm{SiO} 2$ en $\mu \mathrm{M}$ ) dans la baie de Cintra (Octobre 2015 et Mai 2016)

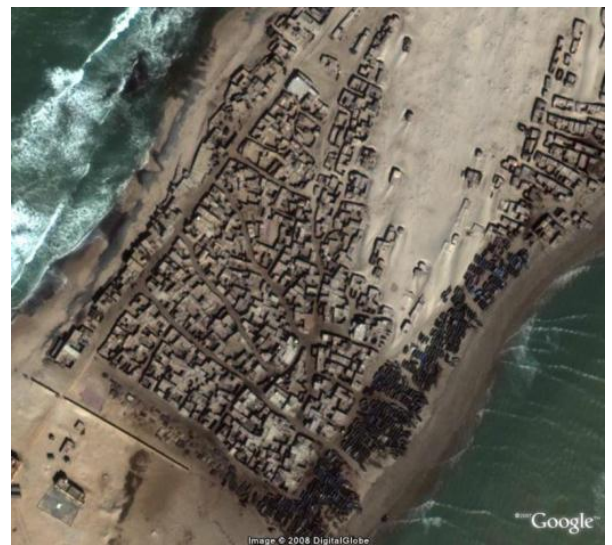

Photo 2:- Ancien Village de pêche de la baie de Cintra (2008)

En effet, Nous avons noté la présence des eaux relativement froides à la côte qui se manifestent sur les distributions en surface des isothermes qui montrent la présence des eaux relativement froides $\left(20^{\circ} \mathrm{C}\right)$ et moins salées $(36.25 \mathrm{psu})$ dues principalement à l'advection des eaux et marquant une activité des résurgences d'eau profonde aussi bien en octobre au sud de cintra qu'en mai au nord (fig. 10). Les profils verticaux de la colonne d'eau le long de la radiale $23^{\circ} \mathrm{N}$ au large de la baie de Cintra indiquent une stratification verticale au large pendant les deux mois favorisant une rétention des eaux riche en matière minérale favorable à la productivité du milieu.

En effet, l'environnement marin de la zone sud de la côte atlantique marocaine est favorable à la ponte de plusieurs espèces à cause de sa haute productivité (Abdellouahab et al., 2016) due à l'influence permanente des résurgences d'eau profonde. Ainsi que la distribution en surface de la chlorophylle et de la fluorescence extraites des images satellites (Behrenfeld, M. J., 2009 ; MODIS-Aqua- 4km) indiquent une richesse biologique importante qui alimente toute la zone côtière pendant le mois d'octobre qui peut être expliquer par l'activité importante des résurgences 
côtières au niveau de cette zone pendant cette période. Alors qu'en mois de mai, les résurgences sont limitées au nord au niveau de Cap Boujdor et la richesse est moins importante au niveau de la zone de Cintra (fig. 11).
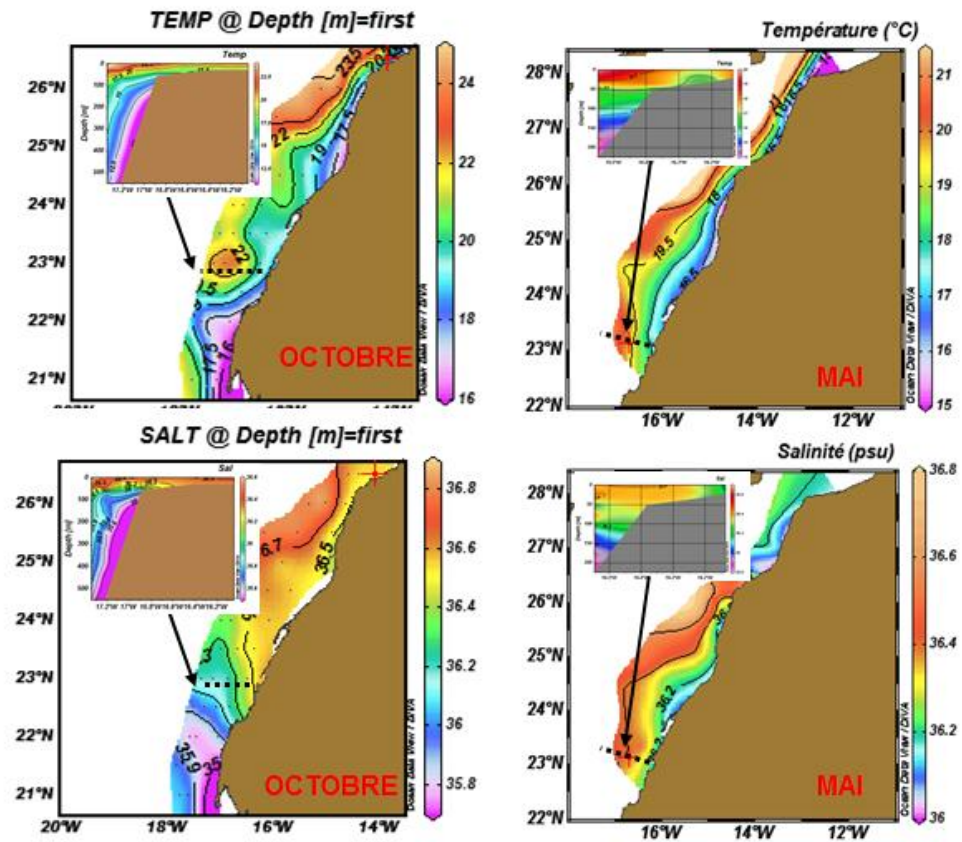

Figure 9:- Distributions superficielle et verticale de la température $\left({ }^{\circ} \mathrm{C}\right)$ et la Salinité (Sal psu) au niveau de la radiale $23^{\circ} \mathrm{N}$ (Atlantida - Octobre 2015 et AMA - Mai 2016)

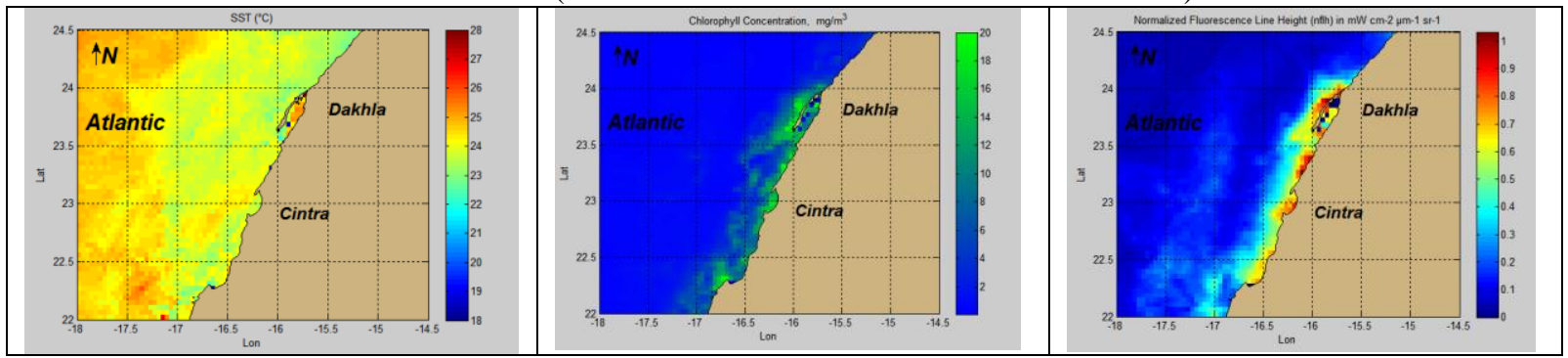

October 2015

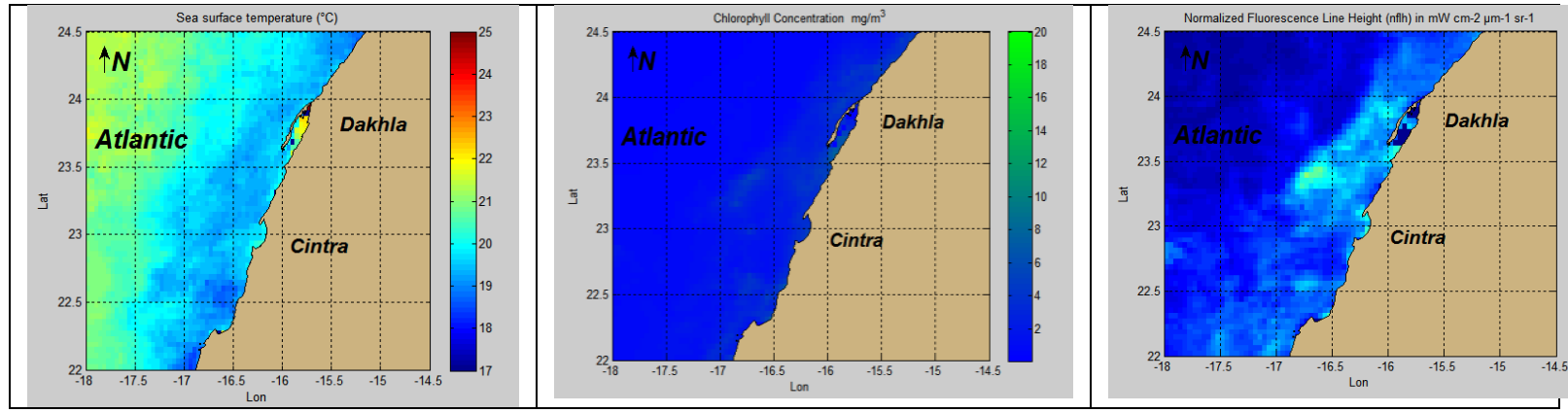

May 2016

Figure 11:- Distributions of SST, Chl-a and Fluo : Octobre 2015 et May 2016

Ainsi, la baie de Cintra se caractérise par une variabilité spatiale et saisonnière de son écosystème marin qui est soumis à l'influence du phénomène d'upwelling de la côte atlantique de la zone sud marocaine. En effet, en présence d'activité d'upwelling (cas du mois d'octobre), la baie s'alimente en eau froide et moins salée et riche en matière minérale favorisant une richesse importante en pigment chlorophyllien dépassant $4 \mu \mathrm{g} / \mathrm{l}$ (fig. 12). Les eaux marines du littoral atlantique se localisent à l'entrée de la baie et peuvent s'approcher de la côte au niveau du cœur de la baie et au sud. En cas d'activité relativement faible des résurgences d'eaux profondes (cas du mois de mai), les 
eaux marines sont moins riches en nutriments se répercutant sur la baie par des faibles taux en pigment chlorophyllien ne dépassant pas $1 \mu \mathrm{g} / \mathrm{l}$.
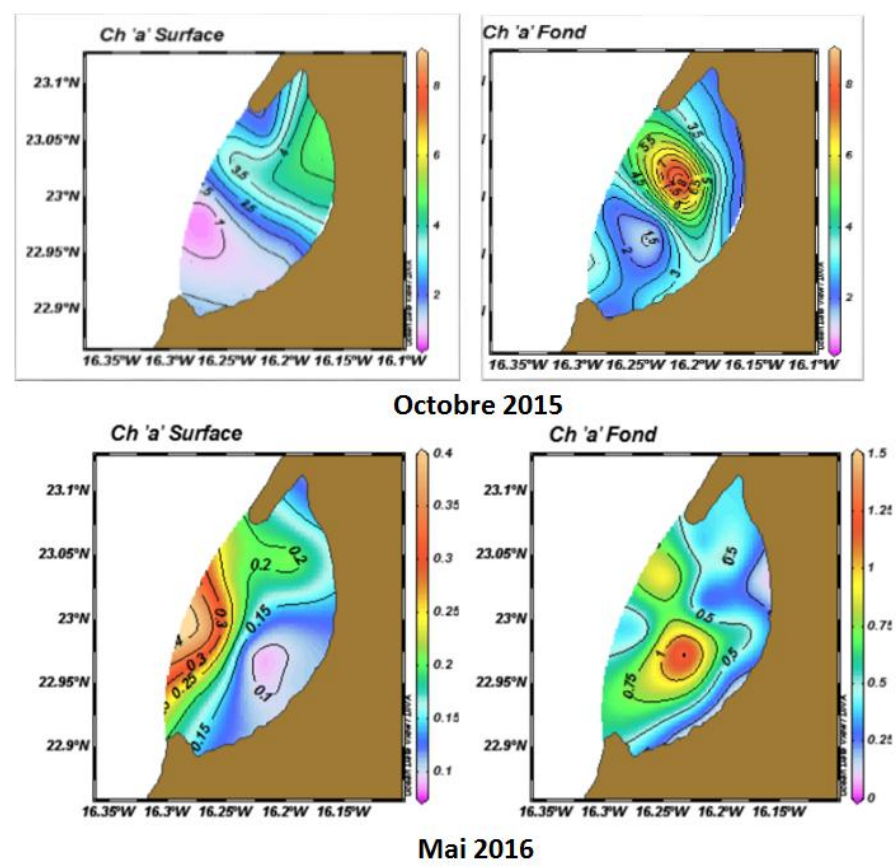

Figure 12:- Distribution en surface et au fond de la chlorophylle ( $\mu \mathrm{g} / \mathrm{l})$ dans la baie de Cintra (Octobre 2015 et Mai 2016)

\section{Conclusion Générale:-}

La baie de Cintra se caractérise par une variabilité saisonnière de son écosystème marin influencée en grande partie par l'état de l'activité du phénomène d'upwelling de la côte atlantique de la zone sud marocaine. En effet, cette zone se caractérise d'une part, en été par un front thermo halin marqué par l'interface entre des eaux de résurgence localisé au niveau de cap Boujdor qui dérive vers le sud de la zone de Dakhla - Cintra et des eaux centrales sud atlantique chaudes, riches en matière minérale et d'autre part, en automne par les résurgences d'eau profondes localisées au sud de Dakhla et sont plus productive. Ainsi la zone d'étude et par suite la baie de Cintra est une zone de rétention des eaux d'upwelling et se trouve en alimentation permanente des eaux enrichies en matière minérale favorisant une productivité primaire importante grâce aux courants de marée qui ont une direction NNE (flux) favorisant l'entrée des eaux marines vers le nord dans la baie et poursuivant leur trajet vers une direction SSW (reflux) en allongeant toute la côte de la baie.

Une fois dans la baie, ces eaux sont soumises au processus de l'évaporation qui représente un deuxième facteur d'enrichissement de la baie notamment au niveau de la zone nord abritée par la pointe du nord où le plateau est plus large et peu profond en s'allongeant vers la sebkha.

Cependant, des apports d'eau déficitaires en oxygène dissous peuvent se manifester exceptionnellement d'une année à l'autre et dépendent de l'intensité du Contrecourant Nord Equatoriale présent principalement en été et de l'activité des résurgences au sud de Dakhla en automne. Ces facteurs dépendent sans doute de l'effet du changement climatique qui se manifeste momentanément au niveau des zones côtières de l'atlantique sud du Maroc.

Toutefois, la baie de Cintra reste par excellence un site très potentiel pour l'aménagement en aquaculture du moment que les sites très protégés favorables à l'aquaculture traditionnelle sont peu nombreux et sont déclassés pour des questions environnementales (lagunes Oualidia, lagune Marchika,..) et d'autres sont menacés (Baie de Dakhla) ; 


\section{Bibliographie:-}

1. AFNOR (1994). Qualité des sols. Recueil des Normes Française. 250 p.

2. ANDA 2015. Appel à Manifestation d'Intérêt, (2015) - Développement de projets d'aquaculture marine dans la région de dakhla oued Eddahab. Agence Nationale pour le développement de l'Aquaculture, p. 104.

3. ABDELOUAHAB Hinde, BERRAHO Amina, BAIBAI Tarik, AGOUZOUK Aziz, MAKAOUI Ahmed, RRHIF Ahmed, 2016: Autumn larval fish assemblages on NWAA coast. Chinese Journal of Oceanology and Limnology. http://dx.doi.org/10.1007/s00343-017-5302-7.

4. Barton E.D., 1998. Eastern boundary of the North Atlantic: Northwest Africa and Iberia. In: Brink KH and Robinson AR (eds) The Sea, The Global Coastal Ocean: Regional Studies and Syntheses, vol. 11.

5. Behrenfeld, M. J., Westberry, T. K., Boss, E. S., et al. (2009). Satellite-detected fluorescence reveals global physiology of ocean phytoplankton. Biogeosciences 6, 779-795.http://dx.doi.org/10.5194/bgd-5-4235-2008. Centre d'Expertise en Analyse Environnementale du Québec et Ministère de l'Agriculture, des Pêcheries et de l'Alimentation du Québec (2003). Détermination de la matière organique par incinération : méthode de perte de feu (paf), ma. 1010-paf 1.0, Ministère de l'Environnement du Québec, 2003, 9 p.CEAEQ, 2003.

6. Direction des Ports et du Domaine Public Maritime (DPDPM, 2013) - Annuaire des côtes marocaines. Ministère d'Equipement et de Transport, p. 14. (http://www.mareespeche.com)

7. FAO, 2010. Report of the FAO working group on the assessment of small pelagic fish off 2 Northwest Africa, Nouakchott, Mauritania, 21-30 April 2009. FAO Fisheries and Agreculture 3 Report, No. 965 Rome, FAO. 252pp.

8. Folk R. L. (1974 modifiée). Brazos river bar: a staday in the significance of grain size parameters. Journ. Sedim. Petrol. Vol. 27, n 1, pp. 3-26.

9. Foucault et Raoult (1988). Dictionnaire de Géologie. Edition Masson. 352 p.

10. Goudet Jean-Luc, 2008. Les OMZ, zones de minimum d'oxygène, s'agrandissent dans l'océan mondial, FuturaSciences ;

11. Hagen E., C. Zûlicke, R. Feistel, 1996. Near-surface structures in the Cape Ghir filament off Morocco, Oceanol. Acta 19 (6) pp. 577-598.

12. Hilmi Karim, Makaoui Ahmed, Ettahiri Omar, Idrissi Mohamed, Abdellaoui Benyounes, Aziz Agouzouk, Tarik BaiBai, Ait Chattou ElMustafa, Ismail Bessa, Bouksim Hassan, 2017. Circulation Marine de la Baie de Cintra (Sud du Maroc) par Modèle Hydrodynamique 2D. European Scientific Journal April 2017 edition Vol.13, No.12 ISSN: 1857 - 7881 (Print) e - ISSN 1857- 7431.

13. Makaoui A. 2008. Etude hydrologique de l'upwelling côtier marocain et sa contribution à la sédimentologie du plateau continental. Thèse nationale à la Faculté des Science Ben M'Sik / Casablanca.

14. Makaoui A., Orbi A., Hilmi K., Zizah S., Larissi J. \& M. Talbi 2005. L'upwelling de la côte Atlantique du Maroc entre 1994 et 1998. C. R. Geoscience, 337: 1518-1524.

15. Makaoui A., A. Orbi, J. Arestigui, A. Ben Azzouz, J. Laarissi, A. Agouzouk, K. Hilmi. Hydrological seasonality of cape Ghir filament in morocco, Vol.4, No.1, 5-13 (2012) Natural Science doi:10.4236/ns. 2012.41002.

16. Makaoui, A. Agouzouk, J. Larissi, T. Baibai, M. Idrissi, I. Bessa, S. Obad, R. Essamoud, et K. Hilmi (2016). Etude hydrodynamique de l'écosystème marin de la baie de Cintra (Maroc). 8ème Conférence Internationale d'Hydrodynamique Marine, 27-28 Octobre 2016, Casablanca, Maroc.

17. Minas, H.J., L.A. Codispoti and R.C. Dugdale, 1982. Nutrients and primary production in the upwelling region off Northwest Africa. Rapp. P.-v. Réun. Cons. int. Explor. Mer 180, pp. 148-183.

18. ORBI A., DAFIR J.E. et BERRAHO A. (1995) -Etude Pluridisciplinaire de la Baie de Dakhla. Trav. et Doc. (ISPM) N86, Décembre 1995, 101p. SHOM. Site web (www.shom.fr).

19. Stramma L, Johnson G. C., Sprintall J. \& Mohrholz V. (2008). Expanding oxygen-minimum zones in the tropical oceans. Science, 320, 655-658.

20. Sogreah (1983)-Rapport de l'étude hydraulique des futures installations du port d'Ad Dakhla. Marché 29, DP82 Direction des ports secondaires, Rabat.

21. Sogreah (1984)- Etude sédimentologique de la baie d'Ad Dakhla, direction des ports Secondaires, Rabat, p. 31.

22. Pelegri J.L, J. Aristegui, L. Cana, L. Gonzalez-Davila, A. Hernandez-Guerra, S. Hernandez-Leon, A. MarreroDiaz, M.F. Montero P. Sangra and M. Santana-Casiano, 2005b. Coupling between the open ocean and the coastal upwelling region off Northwest Africa: water recirculation and offshore pumping of organic matter. Journal of Marine System 54, pp. 3-37.

23. Plumb, R.A.H. (1981). Procedures for handling and chemical analysis of sediment and water samples. Tech. Rep. EPA/CE-81-1. US Army Corps of Engineers. Vicksburg, Va.

24. Wauthy B., 1983. Introduction à la climatologie du golfe de Guinée. Océanoqr.trop., 18(2), pp.103-38. 